\title{
Mechanical Anisotropy and Grain Interaction in Recrystallized Aluminum
}

\author{
C.N. TOMÉ, R.A. LEBENSOHN, and C.T. NECKER
}

Axial compression tests up to 50 pct deformation were performed on rolled and fully recrystallized aluminum sheet stock (AA5754, AA5182, and AA6016) in the direction perpendicular to the sheet. Textures were measured using both X-rays and orientation imaging microscopy (OIM). In all three cases, a systematic in-plane anisotropy was observed, with more strain taking place in the transverse than in the rolling direction. Previous attempts to simulate this in-plane anisotropy for AA5754, starting from the X-ray initial textures and using a one-site polycrystal model, resulted in predictions of more deformation along the rolling than along the transverse direction. An analysis of the OIM textures indicates that there is a nonrandom spatial correlation of the recrystallization and retained rolling components. As a consequence, we implemented grain interaction and co-rotation in a viscoplastic self-consistent (VPSC) polycrystal model, in order to be able to account for orientation correlations. Such an approach allows us to describe the large- and small-angle misorientation distributions, as a function of deformation and to compare them with the available experimental evidence. Concerning the in-plane anisotropy, we conclude that it is very sensitive to details in the texture representation, rather than on grain interactions. Grain-interaction and co-rotation effects, however, have the effect of inducing less severe deformation textures, which is in better agreement with the experimental evidence.

\section{INTRODUCTION}

THE formability of metallic sheet product is often optimal when the amount of in-plane anisotropy is minimized, and this can be accomplished by tailoring the texture. During forming of the sheet the initial texture evolves, often yielding a material that is not mechanically isotropic in-plane. The out-of-plane and in-plane anisotropy of the sheet are experimentally measured by in-plane tensile tests and by throughthickness compression tests, respectively. In the latter case, the anisotropy is measured by the degree of ovalization of originally cylindrical samples.

Polycrystal models permit us to investigate the mechanisms responsible for the evolution of mechanical properties, anisotropy, and texture with deformation. Although most polycrystal models qualitatively reproduce mechanical properties and textures of fcc aggregates, minor variations in initial texture intensities can lead to different texture evolutions, ${ }^{[1]}$ and predicted textures are often sharper than the experimental ones. In addition, the anisotropy of the mechanical response is not always correctly predicted by the models. The reasons can be twofold: either the assumptions on which the model is based are not appropriate/sufficient, or the representation of the texture does not contain the degree of detail required to capture the more subtle characteristics of the material response. We will show here that both aspects of the problem need to be accounted for in order to explain the in-plane anisotropy observed in recrystallized aluminum.

In a previous attempt to predict the behavior of the AA5754 alloy, ${ }^{[2]}$ a one-site viscoplastic self-consistent

C.N. TOMÉ and C.T. NECKER, Technical Staff Members, are with the Materials Science and Technology Division, Los Alamos National Laboratory, Los Alamos, NM 87545. Contact e-mail: tome@lanl.gov R.A. LEBENSOHN, Long Term Visiting Staff Member, Materials Science and Technology Division, Los Alamos National Laboratory, is on leave from the National University of Rosario, 2000 Rosario, Argentina.

Manuscript submitted September 24, 2001. $(\text { VPSC })^{[3]}$ simulation was carried out, using a discretized form of the measured X-ray initial texture as input. This calculation predicted an in-plane anisotropy after $50 \mathrm{pct}$ compression which was reversed with respect to the measured one. This conclusion remained unaltered whether or not the grain-shape evolution was accounted for and for different degrees of grain-matrix interactions (full constraint, relaxed constraints, self-consistent secant, and selfconsistent tangent). Such a discrepancy between the experiment and predictions motivated the more comprehensive analysis of the present work.

For this study, through-thickness compression tests (i.e., along the normal direction (ND) of the sheet) up to about 50 pct strain were performed on O-tempered AA5754 and AA5182 and T4-treated AA6016 alloys. In all three cases, the same in-plane anisotropy was observed, namely, a slight tendency to ovalization with the major and minor axes along the transverse direction (TD) and rolling direction (RD) of the sheet, respectively. This anisotropy, although small, is reproducible. The suspicion was that a nonrandom spatial correlation of the various retained rolling components might exist in the initial texture, and that grain interactions may tip the balance and determine the evolution of in-plane anisotropy. If orientation correlations were the reason for the disagreement between the model and experiment reported in Reference 2, then both the model and the experimental technique used to characterize initial textures should be upgraded. Therefore, in the present work, the initial textures were measured by means of orientation imaging microscopy (OIM), a technique which provides relevant information about the orientation correlations. Eventually, this information was used as input for two-site VPSC simulations, ${ }^{[4]}$ in order to capture the effect of grain interactions and orientation correlations on the resulting textures and in-plane anisotropy. We show subsequently that this approach reproduces the anisotropy measured in the three alloys, predicts a slower 
Table I. Composition of the Three Al Alloys (Units of Weight Percent)

\begin{tabular}{cccccc}
\hline Alloy & $\mathrm{Mg}$ & $\mathrm{Mn}$ & $\mathrm{Fe}$ & $\mathrm{Si}$ & $\mathrm{Cu}$ \\
\hline AA5754 & 3.06 & 0.37 & 0.13 & - & - \\
AA5182 & 4.85 & 0.36 & 0.15 & - & - \\
AA6016 & 0.40 & 0.20 & 0.50 & 1.20 & 0.20 \\
\hline
\end{tabular}

evolution of deformation textures (more in line with experimental observations), and predicts small-angle misorientation distributions which are also consistent with experimental evidence. Central to the model are the concepts of interaction and co-rotation between orientations.

The plan of this article is as follows. In Section II, we describe the experimental procedures, i.e., mechanical testing and texture measurements, used to characterize the aluminum samples. In Section III, we present the main features of the two-site VPSC formulation and the co-rotation scheme adopted for texture updating and describe how the measured OIM texture is processed to represent the correlations found in the initial polycrystal. In Section IV, we compare the predicted and experimental textures and in-plane anisotropy. We also discuss the predicted evolution of misorientations. Finally, the conclusions are presented in Section V.

\section{EXPERIMENTAL PROCEDURE}

\section{A. Mechanical tests}

The aluminum samples used in this study were the magnesium-manganese-iron alloys AA5754, AA5182, and AA6016. Table I shows the composition of these three alloys. The material was Direct Chill cast, conventionally processed sheet stock in the recrystallized condition. The AA5754 sheet was 1.47-mm thick, while the AA5182 and AA6016 sheets were $1.00-\mathrm{mm}$ thick.

The compression tests were performed on a screw-driven Instron model 1125 apparatus. All tests were run at a strain rate of $10^{-3} \mathrm{~s}^{-1}$. The platens were lubricated with Dow Corning (Midland, MI) Molykote 321R. Disks of $6 \mathrm{~mm}$ (AA5754) and $10 \mathrm{~mm}$ (AA5182 and AA6016) in diameter were electro-discharge machined. The compression samples were built by stacking five disks (AA5754) or ten disks (AA5182 and AA6016) and holding them together with a rubber sleeve while keeping the RD aligned in the sample. A "cup" was machined in the top and bottom disks of the stack, with the purpose of retaining the lubricant throughout deformation. The cup was $0.08-\mathrm{mm}$ deep, and the outer rim was $0.51-\mathrm{mm}$ wide. These dimensions were empirically optimized in order to minimize friction, while avoiding triaxial stress states in the sample. Sample fabrication and stacking were performed without compromising the sheet surface and preserving the RD alignment. Following testing, the compression data were corrected for the load-frame stiffness, and the true stress-strain response was calculated. Final strains were between 40 and 50 pct for the different samples (Table II). The stress-strain response of the three alloys is depicted in Figure 1. In all three cases, we measured a reproducible inplane anisotropy reported in Table II, which manifests itself as a slight ovalization of the disks, with the TD deforming more than the RD. The ovalization measurements were done by averaging the RD and TD diameters of the interior disks
Table II. Compression along the ND; Ovalization Measured on Different Samples for the Three Al Alloys

\begin{tabular}{ccccc}
\hline Alloy & $\varepsilon_{N D}$ & $\varepsilon_{T D}$ & $\varepsilon_{R D}$ & $\varepsilon_{T D} / \varepsilon_{R D}$ \\
\hline AA5754 & 0.4940 & 0.2530 & 0.2410 & 1.049 \\
& 0.4817 & 0.2442 & 0.2375 & 1.028 \\
& 0.4897 & 0.2520 & 0.2377 & 1.060 \\
AA5182 & 0.6020 & 0.3029 & 0.2991 & 1.013 \\
& 0.4019 & 0.2095 & 0.1924 & 1.089 \\
& 0.4036 & 0.2097 & 0.1939 & 1.082 \\
AA6016 & 0.4498 & 0.2352 & 0.2146 & 1.096 \\
& 0.4736 & 0.2445 & 0.2291 & 1.067 \\
& 0.4316 & 0.2199 & 0.2118 & 1.038 \\
& 0.3968 & 0.2010 & 0.1958 & 1.027 \\
\hline
\end{tabular}

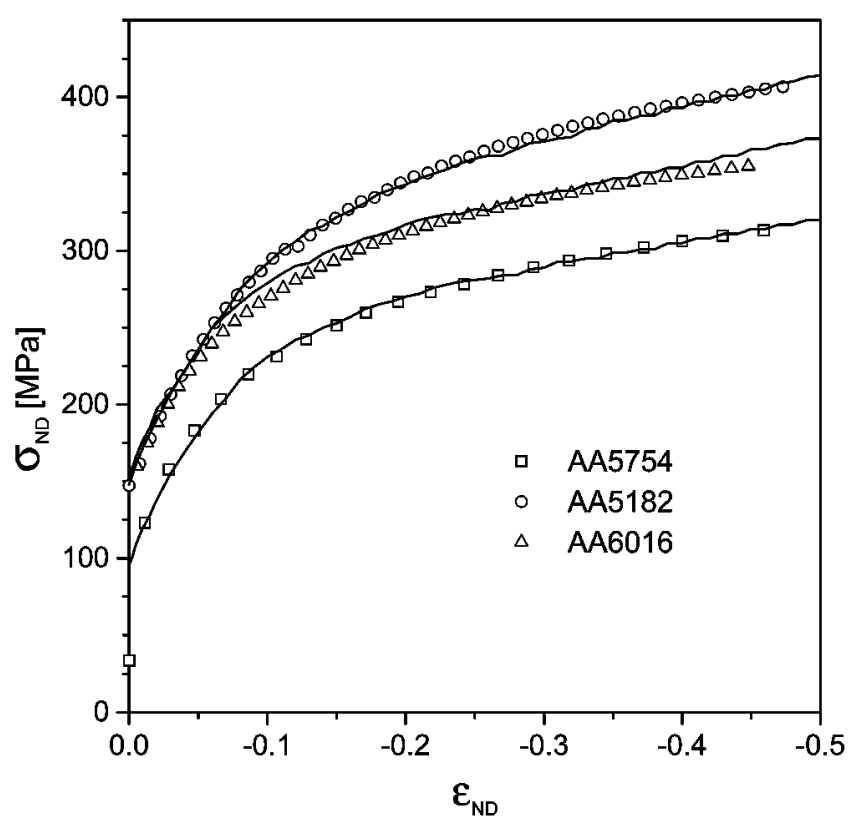

Fig. 1-Experimental (symbols) and simulated (lines) stress-strain response for the three $\mathrm{Al}$ alloys tested in compression at a rate of $\varepsilon=10^{-3} \mathrm{~s}^{-1}$.

of each sample. The top and bottom disks (which include the cup) were not included in the averaging, because their deformation may be affected by friction or triaxiality effects.

In addition to the compression tests, the AA5754 sheet was also tested in tension along the RD. A sample machined to ASTM E-8 specifications was strain-gaged for measuring the axial and width strain, and the through-thickness strain was inferred from the latter two components. The hardening response is shown in Figure 2, together with the throughthickness normal direction (ND) and TD strain evolution. The out-of-plane anisotropy of the sheet is evident from the divergence between the ND and TD strain components. The serrations in the lateral strains are due to a Luders-type propagation of the tensile deformation, which the lateral strain gage records intermittently as it passes along the axis of the sample.

\section{B. The X-Ray Texture Measurements}

The initial textures of the three Al sheets were measured by X-ray diffraction and by OIM. The final compression textures were measured using X-rays only, since the quality 


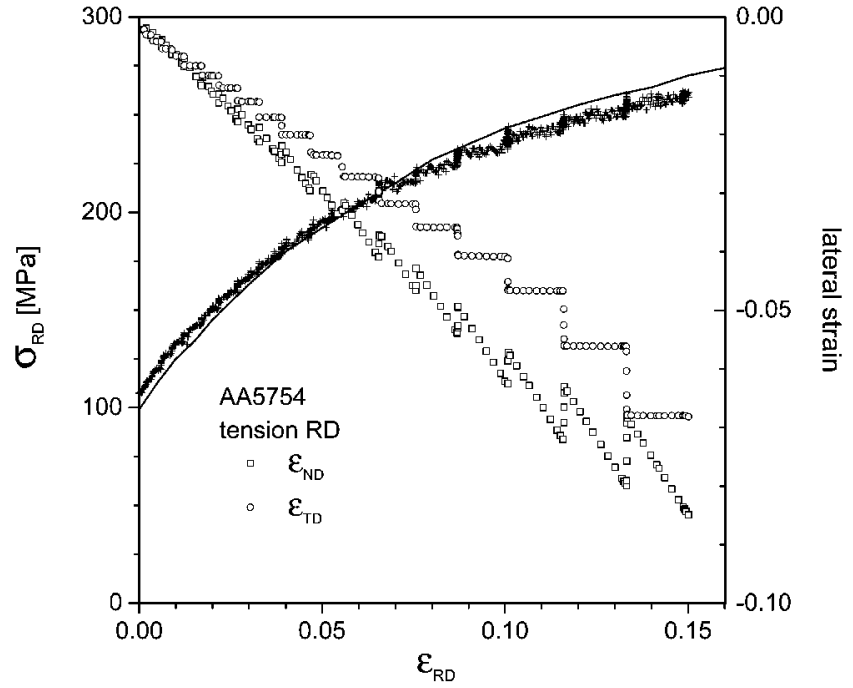

Fig. 2-Experimental and simulated stress-strain curves and experimental lateral strains for a tensile test along the RD of the AA5754 alloy.

of the OIM patterns deteriorates in heavily cold-worked samples. The samples for texture analysis of the as-received material were fabricated by cutting and stacking the sheet for through-thickness analysis. Compressed disks were also arranged in a metallographic mount for through-thickness analysis in the ND-RD plane, with the RD alignment preserved. The samples were ground, polished, and etched. The X-ray texture was measured by the standard reflection technique on a Scintag five-axis pole-figure goniometer. The raw data were corrected and orientation distribution functions ODFs were calculated using popLA software. ${ }^{[5]}$ The ODF intensities, given at 5 deg intervals, were integrated over 5 and 10-cells to produce a "full" discrete set of 23,328 orientations and a "reduced" set of 2916 orientations, respectively. The cell integration provides a weight which represents the volume fraction of each orientation in the texture. The (111) pole figures of the three recrystallized aluminum alloys are shown in Figure 3 for the full and the reduced representations. The relatively low intensities indicate that the material is only mildly textured as a consequence of the recrystallization process. The near coincidence between the full and the reduced representations suggests that we use the reduced texture for predicting the mechanical response of the aggregate. We verified in this work that both give practically identical results.

\section{The OIM Texture Measurements}

Microtexture measurements were conducted using TexSem Lab's OIM instrumentation on a PHILIPS* XL30 fieldemission gun-scanning electron microscope (Reference 6 provides a system description). The same samples used to measure the X-ray textures were used for these measurements, except that the surface was electropolished. The system was set up to scan an area of approximately $500 \times$ $1000 \mu \mathrm{m}$, with steps of $2.5 \mu \mathrm{m}$. The resulting data included the orientation as a function of position. Grain sections were defined by a minimum $15 \mathrm{deg}$ boundary misorientation between neighboring pixels, and grain sizes were assumed to be proportional to the number of pixels within the section.
Figure 4 shows a portion of the orientation map corresponding to the as-received AA5754, in which the grains appear arbitrarily shaded. A program was written to identify and build a table of nearest neighbors to each grain. Such information was used as input in the modeling part of this work (Section III-D).

A total of 2215, 4234, and 1579 different orientations (grains) were measured by OIM for the AA5754, AA5182, and AA6016 alloys, respectively. Weights proportional to the area of the grain's section were assigned to each orientation in order to generate an "OIM texture file." In Figure 3, we compare the OIM and X-ray (111) pole figures for the three alloys. It can be seen that the OIM and the X-ray pole figures are qualitatively similar, with the OIM ones being slightly more intense. It will be shown later that these differences have a quantitative effect on the predicted inplane anisotropy of the aggregates. This, in turn, raises the question of which technique provides a more realistic representation of the actual texture. The OIM measurements provide direct information on the crystallographic orientations and their surface fractions, but a sufficiently large number of orientations need to be measured using a step much smaller than the grain size. The X-ray diffraction, on the other hand, has the capability of sampling larger areas, but the ODF that follows from the analysis of pole figures is affected by the numerical procedures used to deconvolute the information and complete the unmeasured rim of the pole figure. In addition, the X-ray texture provides no information about possible orientation correlations in the aggregate. As a consequence, OIM textures are better suited for analyzing effects that may depend subtly upon details of the texture and orientation correlations.

The discretized textures extracted from the OIM measurements and used in the simulations are identical to the measured ones, since all the orientations measured were used for representing them. By postprocessing these OIM measurements, a number of neighbor orientations (ranging from one to 12 ) were assigned to each grain. Only those grains whose boundaries were entirely within the measured area were counted as neighbors. With this topological information, we built sets of orientation pairs consisting of 6134,12294 , and 4493 pairs for the AA5754, AA5182, and AA6016 alloys, respectively. In each set, a given orientation was represented as many times as the number of neighbors it had, and the associated volume fraction was split accordingly to account for this multiplicity. These "paired" discrete textures are identical to the measured ones shown in Figure 3.

\section{Statistics on Rolling Components}

The three aluminum samples studied here are fully recrystallized and, as a consequence, the texture exhibits "cube" and "rotated cube" components. In addition, the aggregate retains some of the rolling-texture components i.e., "Goss," "brass," "copper," and "S" (Table 3 provides definitions). We want to establish whether the proportion and the spatial correlation of these components could be responsible for the observed mechanical behavior. As a consequence, we processed the discretized X-ray and OIM textures in order to identify the fraction of each component and, in the OIM case, to characterize the spatial correlations between components. A criterion of a $15 \mathrm{deg}$ misorientation with respect to the ideal component is used to assign a given orientation 


\section{full X-ray
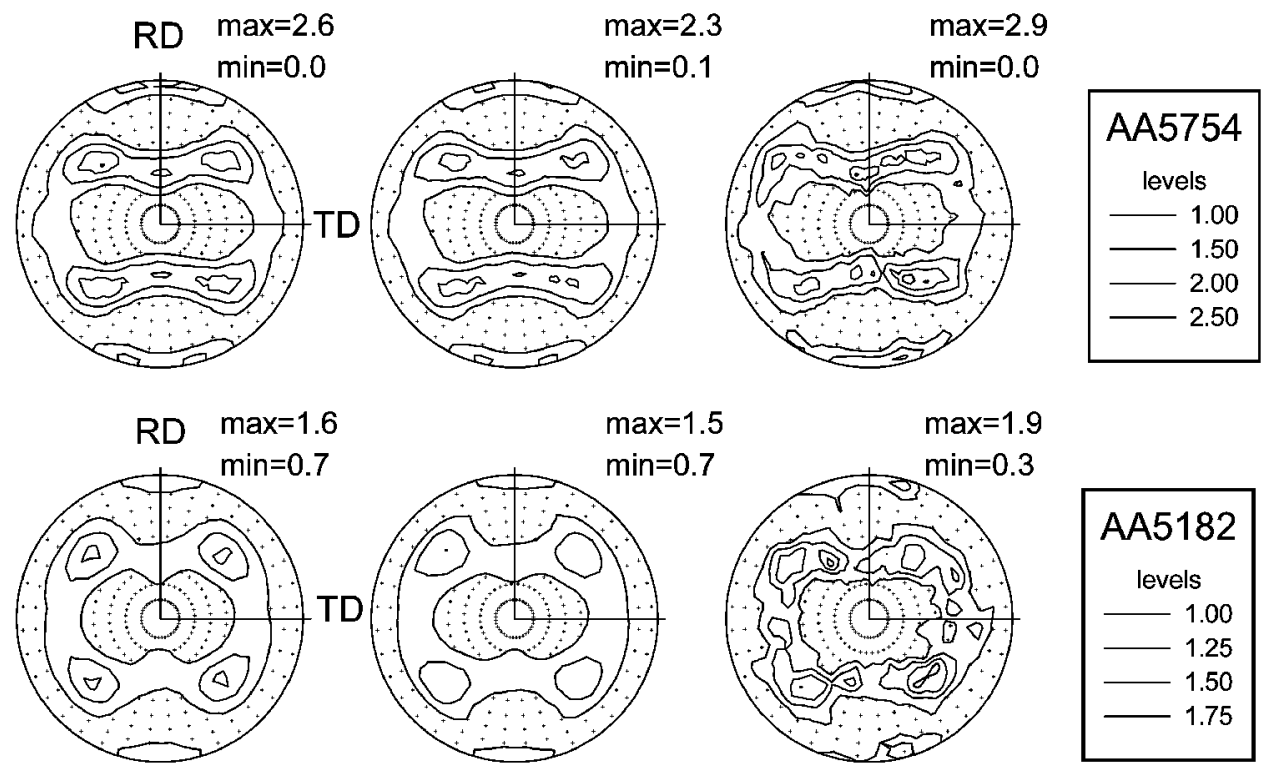

$\max =1.5$
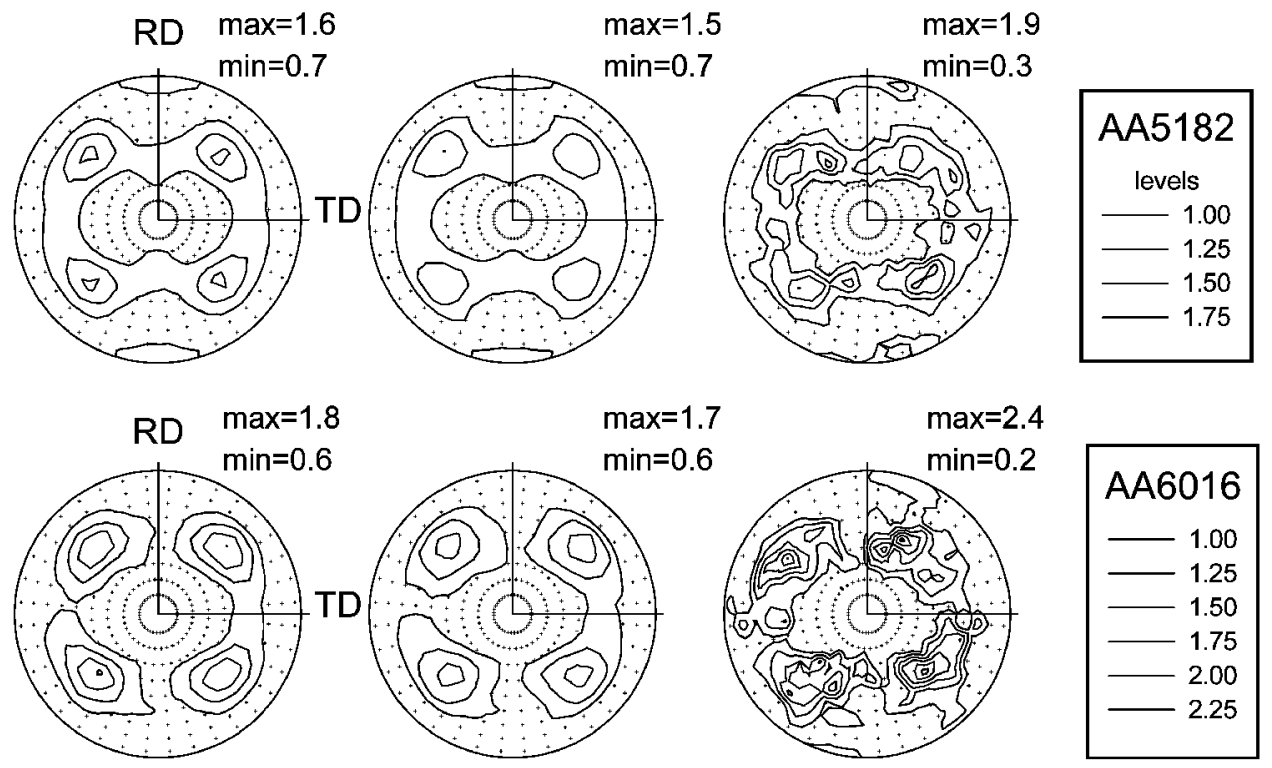

Fig. 3-Initial (111) pole figures for the three rolled and recrystallized aluminum alloy textures considered here, obtained via X-ray or OIM measurements. "Full X-ray" corresponds to using all 23,327 intensities in the ODF, "reduced X-ray" refers to a reduced texture representation using 2916 orientations, and "OIM" plots all the orientations measured by electron backscattering.

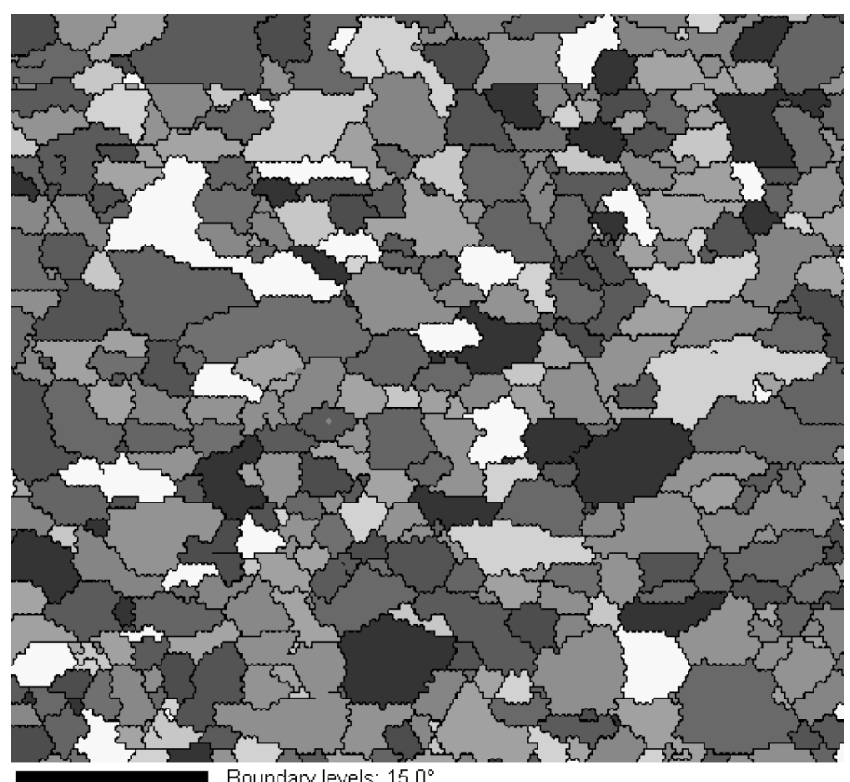

$75.00 \mu \mathrm{m}=30$ steps Grain Color

Fig. 4-Microstructure (RD-ND plane) of the AA5754 alloy (only a fraction of the actual OIM scan is shown here). to that component. The components considered here and the initial volume fraction that they represent in each of the aluminum samples tested are summarized in Table III. Observe that the statistics differ little for the X-ray and the OIM textures, with $\mathrm{X}$-rays being associated with a systematically smaller fraction of cube and rotated cube components than OIM. The most noticeable differences are that the fraction of rotated cube, copper, and S components is substantially larger and the amount of "random" components is substantially smaller for AA5754 than for the other two alloys. As a consequence, the anisotropy of AA5754 turns out to be qualitatively different than that of the AA5182 and AA6016 alloys.

With regard to performing a statistic on spatial orientation correlations, only the OIM texture can be used to such an effect. We scanned the OIM, identified all the grains belonging to a particular component, and, for each component, classified the nearest neighbors into one of the components listed in Table III and accumulated the correlated volume fractions. If the orientations were spatially distributed in a random fashion, the probability of finding as a first neighbor a grain associated with a particular component would simply be equal to the volume fraction represented by such a component. However, an analysis of the OIM information shows that there is a nonrandom spatial correlation between the 
Table III. Volume Fraction (within 15 Deg Misorientation from the Ideal) of Recrystallized and Retained Rolling Components for the Three Al Alloys Analyzed Here; Calculated from the OIM Texture and from the Discretized ODF Measured by X-ray Diffraction

\begin{tabular}{|c|c|c|c|c|c|c|c|c|c|}
\hline \multirow[b]{2}{*}{ Component } & \multirow[b]{2}{*}{$\varphi_{1}$} & \multirow[b]{2}{*}{$\Phi$} & \multirow[b]{2}{*}{$\varphi_{2}$} & \multicolumn{2}{|c|}{ AA5754 } & \multicolumn{2}{|c|}{ AA5182 } & \multicolumn{2}{|c|}{ AA6016 } \\
\hline & & & & OIM & X-Ray & OIM & X-Ray & OIM & X-Ray \\
\hline Cube $\{001\}\langle 100\rangle$ & 0 & 0 & 0 & 7.1 & 6.9 & 6.2 & 4.4 & 5.5 & 5.2 \\
\hline Rot cube & 0 & 22.5 & 0 & 11.0 & 9.3 & 5.9 & 4.3 & 7.8 & 4.9 \\
\hline Goss $\{011\}\langle 100\rangle$ & 0 & 45 & 0 & 1.7 & 2.4 & 2.1 & 2.1 & 1.6 & 1.7 \\
\hline Brass $\{110\}\langle 112\rangle$ & 35.3 & 45 & 0 & 6.7 & 5.8 & 4.3 & 4.0 & 5.5 & 4.5 \\
\hline Copper $\{112\}\langle 111\rangle$ & 90 & 35 & 45 & 8.4 & 7.0 & 3.8 & 4.9 & 3.1 & 3.6 \\
\hline$S\{123\}\langle 634\rangle$ & 59 & 37 & 63 & 13.3 & 14.5 & 8.6 & 8.1 & 7.3 & 7.1 \\
\hline Balance & - & - & - & 50.8 & 54.1 & 68.1 & 72.3 & 69.2 & 73.0 \\
\hline
\end{tabular}

Table IV. Spatial Correlation Index between the Texture Components Listed in Table II for the Three Al Alloys

\begin{tabular}{lccccccc}
\hline AA5754 & $\mathrm{Cu}$ & $\mathrm{Br}$ & $\mathrm{S}$ & Goss & Cube & RotC & other \\
\hline $\mathrm{Cu}$ & 1.01 & 0.84 & 1.04 & 0.83 & 1.22 & 1.71 & 1.30 \\
$\mathrm{Br}$ & - & 1.21 & 1.20 & 0.97 & 1.03 & 1.11 & 0.94 \\
$\mathrm{~S}$ & - & - & 0.74 & 1.17 & 1.13 & 1.17 & 0.94 \\
Goss & - & - & - & 0.00 & 0.64 & 1.05 & 0.90 \\
Cube & - & - & - & - & 0.86 & 0.89 & 1.02 \\
RotC & - & - & - & - & - & 0.81 & 0.98 \\
balance & - & - & - & - & - & - & 0.89 \\
\hline AA5182 & $\mathrm{Cu}$ & $\mathrm{Br}$ & $\mathrm{S}$ & Goss & Cube & RotC & other \\
\hline $\mathrm{Cu}$ & 0.51 & 1.31 & 0.90 & 1.02 & 1.39 & 1.02 & 0.86 \\
$\mathrm{Br}$ & - & 0.43 & 0.81 & 1.26 & 0.92 & 0.86 & 0.98 \\
$\mathrm{~S}$ & - & - & 0.75 & 0.93 & 1.20 & 0.91 & 0.97 \\
Goss & - & - & - & 0.17 & 0.82 & 1.07 & 0.97 \\
Cube & - & - & - & - & 0.74 & 1.27 & 1.14 \\
$\mathrm{RotC}$ & - & - & - & - & - & 0.96 & 0.97 \\
Balance & - & - & - & - & - & - & 1.01 \\
\hline AA6016 & $\mathrm{Cu}$ & $\mathrm{Br}$ & $\mathrm{S}$ & Goss & Cube & RotC & other \\
\hline Cu & 1.10 & 0.38 & 0.91 & 0.82 & 1.27 & 0.48 & 0.88 \\
Br & - & 0.37 & 1.16 & 2.12 & 1.26 & 0.73 & 1.00 \\
S & - & - & 0.48 & 1.24 & 0.50 & 1.12 & 0.97 \\
Goss & - & - & - & 2.18 & 0.80 & 0.20 & 1.07 \\
Cube & - & - & - & - & 0.91 & 0.51 & 1.07 \\
RotC & - & - & - & - & - & 0.95 & 1.14 \\
Balance & - & - & - & - & - & - & 1.01 \\
\hline
\end{tabular}

components. The ratio between the correlated volume fraction and the volume fraction associated with each component (the one listed in Table III) is a measure of the degree of spatial correlation. The results of such calculations are summarized in Table IV for each of the three materials. Observe that, although it is obvious that in a given material some components exhibit moderate correlation, there is not a common pattern valid for all three aluminum alloys. For example, Goss orientations are unlikely to be contiguous in the AA5754 and AA5182 alloys, while they have a muchlarger-than-random probability of being first neighbors in AA6016.

\section{MODELING}

\section{A. The VPSC Formulation: one-Site and two-Site}

In what follows, we present and discuss some features of the one-site and two-site VPSC formulations relevant to this work. For a comprehensive description of both models, the reader is referred to References 3 and 4 . From here on, we will refer to "grain interaction" only in connection with the two-site model.

The rate-sensitivity equation relating the stress and strain rate at a single-crystal level is given by

$$
\varepsilon=\sum_{s} m^{s} \gamma^{s}=\gamma_{o} \sum_{s} m^{s}\left(\frac{m^{s}: \sigma^{\prime}}{\tau^{s}}\right)^{n}
$$

where $\tau^{s}, m^{s}$, and $\gamma^{s}$ are the critical stress, the Schmid tensor, and the shear rate associated with slip system $(s)$, respectively, $\gamma_{o}$ is a reference strain rate; and $n$ can be loosely regarded as the inverse of the rate-sensitivity of the material. In practice, Eq. [1] is to be regarded more as a way of avoiding ambiguities in the selection of slip systems than as a rigorous description of the rate-sensitivity of slip. The values of $\tau^{s}$ are updated incrementally using an extended Voce hardening law, discussed in Section III-C. The symmetric second-order Schmid tensor associated with a slip system is defined in terms of the normalized slip-plane normal $\left(\bar{n}^{s}\right)$ and slip direction $\left(\overline{\mathrm{b}}^{s}\right)$, as

$$
m_{i j}^{s}=\frac{1}{2}\left(b_{i}^{s} n_{j}^{s}+b_{j}^{s} n_{i}^{s}\right)
$$

The corresponding skew-symmetric tensor associated with a slip system is required in connection with crystallographic rotation and is given by

$$
q_{i j}^{s}=\frac{1}{2}\left(b_{i}^{s} n_{j}^{s}-b_{j}^{s} n_{i}^{s}\right)
$$

Unlike the full-constraints Taylor model, in which the local strain rates in the grains are enforced to be equal to the macroscopic strain rate applied to the polycrystal, the one-site VPSC model allows each grain to deform differently, according to its directional properties and depending on the strength of the interaction between the grain and its surroundings. Each grain is regarded as an ellipsoidal inclusion, surrounded by a homogeneous effective medium (HEM) which has the average properties of the polycrystal. The HEM properties are not known in advance, but have to be calculated as the average of the individual grain behaviors, once convergence is achieved. Clearly, within this approach, the effect of the neighborhood on the mechanical response of each grain is accounted for only in an average way through the interaction between the grain and the HEM. No special consideration is given to the specific neighbors of a given orientation. As a consequence, in the one-site approximation, 
the fluctuations in the mechanical response of different grains (or parts of grains) having the same orientation but different local environments are completely disregarded. Conceived to overcome the limitations of the one-site approximation, the two-site VPSC model considers the interaction of two ellipsoidal inclusions (representing grains or parts of grains) embedded in a HEM, whose properties must be obtained self-consistently as an average of the local properties. In this way, the two-site model is less deterministic than the one-site model, i.e., different grains (or parts of grains) deform differently, influenced by the interaction with a global HEM, but also by its local nearest-neighbor grain (or part of a grain). In both the one-site and two-site formulations, the connection between microscopic and macroscopic magnitudes is given by the so-called interaction equation. In the one-site case, this interaction equation reads as

$$
\tilde{\varepsilon}=-\tilde{M}: \tilde{\sigma}^{\prime}
$$

where

$$
\begin{gathered}
\tilde{\varepsilon}=\varepsilon-\bar{\varepsilon} \\
\tilde{\sigma}^{\prime}=\sigma^{\prime}-\bar{\sigma}^{\prime} \\
\tilde{M}=n^{\mathrm{eff}}(\mathrm{I}-\mathrm{S})^{-1}: S: M^{\mathrm{sec}}
\end{gathered}
$$

Here, $\left(\varepsilon, \sigma^{\prime}\right),\left(\bar{\varepsilon}, \bar{\sigma}^{\prime}\right)$, and $\left(\tilde{\varepsilon}, \tilde{\sigma}^{\prime}\right)$ are the strain-rate and the deviatoric stress tensors in the inclusion, the effective medium, and their corresponding deviations, respectively. The symbol $\tilde{M}$ is the interaction tensor, a function of the viscoplastic Eshelby tensor $(S),{ }^{[7]}$ the macroscopic secant compliance $\left(\mathrm{M}^{\mathrm{sec}}\right)$, and an effective interaction parameter $\left(n^{\mathrm{eff}}\right)$. The latter is equal to the power $n$ of Eq. [1] if the tangent formulation is used ( $n=20$ in our calculations), or $n^{\mathrm{eff}}=1$ if the secant formulation is used. In our calculations, an effective value of $n^{\text {eff }}=10$ is used, which represents an intermediate inclusion-medium interaction. ${ }^{[8]}$ In addition to the strain rate (symmetric component of the velocity gradient), the inclusion formalism also provides the rotation rate (skew-symmetric component of the velocity gradient) as

$$
\tilde{\omega}=W: S^{-1}: \tilde{\varepsilon}
$$

In the two-site formulation, the interaction equation adopts a more complicated form. ${ }^{[4]}$ If the two ellipsoids are identified as 1 and 2, the interaction equation can be written as

$$
\begin{aligned}
& \tilde{\varepsilon}^{1}=-\tilde{M}^{11}: \tilde{\sigma}^{\prime 1}-\tilde{M}^{12}: \tilde{\sigma}^{\prime 2} \\
& \tilde{\varepsilon}^{2}=-\tilde{M}^{21}: \tilde{\sigma}^{\prime 1}-\tilde{M}^{22}: \tilde{\sigma}^{\prime 2}
\end{aligned}
$$

where $\left(\varepsilon^{1}, \sigma^{\prime 1}\right)$ and $\left(\varepsilon^{2}, \sigma^{\prime 2}\right)$ are the local states inside both inclusions; $\tilde{M}^{11}$ and $\tilde{M}^{22}$ are the one-site interaction tensors given by Eq. [5]; while $\tilde{M}^{12}$ and $\tilde{M}^{21}$ are the two-site interaction tensors, which are a function of the shape, relative volume, and orientation of both ellipsoids and the effective compliance of the HEM (explicit expressions are given in Reference 4). As for the rotation rates of the two ellipsoids, they are given by a linear form of the strain-rate deviations that represents an extension of Eq. [6]. ${ }^{[4]}$

$$
\begin{aligned}
& \tilde{\omega}^{1}=-W^{11}: \tilde{\varepsilon}^{1}-W^{12}: \tilde{\varepsilon}^{2} \\
& \tilde{\omega}^{2}=-W^{21}: \tilde{\varepsilon}^{1}-W^{22}: \tilde{\varepsilon}^{2}
\end{aligned}
$$

Once $M^{\text {sec }}$ has been adjusted self-consistently (Reference 3 provides details), in the one-site model, the local states can be calculated by solving the $5 \times 5$ nonlinear system obtained by combination of the constitutive Eq. [1] and the interaction Eq. [4]. In the two-site case, a $10 \times 10$ nonlinear system must be solved by combining the two-site interaction Eqs. [7] and two constitutive equations of the type of Eq. [1], one for each inclusion. The macroscopic state can be then calculated as a weighted average of the so-solved local states.

\section{B. Co-Rotation Scheme}

The way of dealing with the ellipsoid and crystallographic rotations is central to the co-rotation scheme and will be discussed here in some detail. While Eqs. [6] or [8] provide the rotation trend for the ellipsoid that represents the grain, other considerations may require us to modify this trend to make it compatible with the rotation of neighboring grains. In what follows, we will discuss a "grain co-rotation" scheme used to decide the ultimate rotation, independently of whether the rotation rates are derived from a one-site (Eq. [6]) or a two-site (Eq. [8]) calculation. The term "grain interaction," on the other hand, will be associated exclusively with two-site calculations (Eqs. [7] and [8]).

First, let us identify the different rotation-rate (spin) components involved (all the following are skew-symmetric tensors and, therefore, have three independent off-diagonal components).

(1) $\Omega_{i j}$ : The applied macroscopic rotation rate, i.e., the skewsymmetric component of the macroscopic velocity gradient.

(2) $\omega_{i j}^{g}=\Omega_{i j}+\tilde{\omega}_{i j}^{g}$ : The rotation rate of the ellipsoidal inclusion that represents each grain, given by the skewsymmetric component of the local-velocity gradient. Since the velocity gradient is uniform within the domain of the inclusion, this is also the spin of the cavity that contains the inclusion. The deviations from the average $\tilde{\omega}_{i j}^{g}$ values are given by Eqs. [6] or [8], depending on whether a one-site or two-site approach is used, respectively.

(3) $\omega_{i j}^{p, g}=\sum_{s} q_{i j}^{s, g} \gamma^{s, g}$ : The plastic rotation rate of grain $g$ (refer also to Eq. [3]). It describes the rotation of the main axes of the ellipsoid due to crystallographic shear only (i.e., the crystallographic orientation remains invariant).

The following ideal process helps illustrate how these spin components are related. Assume that one describes separately the deformation (stretch) and rotation of the inclusion and of the cavity in which it fits, during one incremental deformation step. The process is sketched in Figure 5, with Figure 5(a) representing the initial state and Figure 5(f) the final state after simple shear deformation. In Figure 5(b), the inclusion has been separated from the cavity before any stretch and rotation is imposed. In Figure 5(c), both have been rotated by $\omega^{g} \Delta t$, without stretching. In Figure 5(d), the stretch $\varepsilon^{g}=\bar{\varepsilon}+\tilde{\varepsilon}$ is enforced onto (the boundary of) the cavity and onto the inclusion. Since the latter deforms by shear in crystallographic planes, the crystallographic orientation remains unchanged, but the main axes of the associated ellipsoid experience a plastic rotation $\left(\omega^{p, g} \Delta t\right)$. As a consequence, a rigid counter-rotation $\left(-\omega^{p, g} \Delta t\right)$ has to be imposed onto the ellipsoidal inclusion for it to fit into the cavity (Figure 5(e)). The overall lattice rotation for the step is $\omega^{\text {lat }, g} \Delta t$, where 


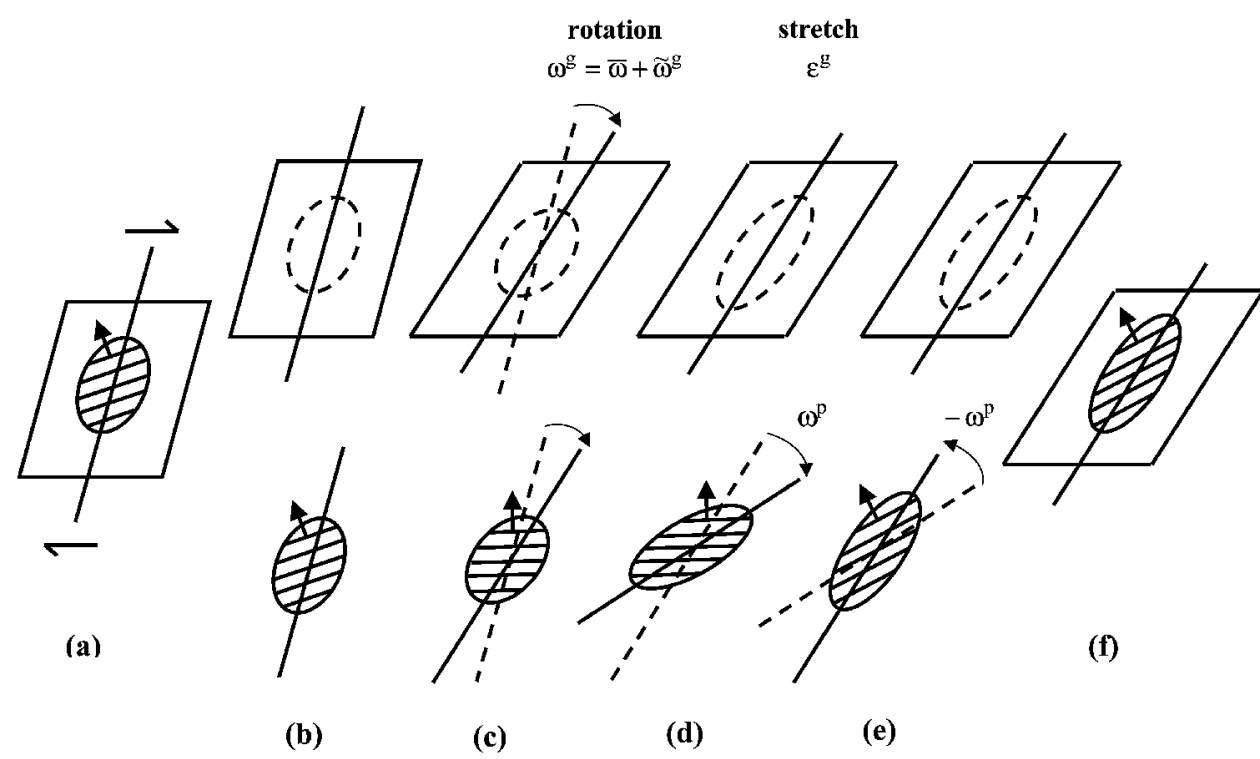

Fig. 5-Idealized sequence illustrating the stretch and rotation of the grain during an incremental deformation step. The displacements of the grain (inclusion) and the cavity in which it fits are described separately: $(a)$ and $(b)$ initial state, $(c)$ rigid rotation of grain and cavity without stretch, $(d)$ stretch of grain and cavity (same) misaligns the main axes of the ellipsoid, $(e)$ counter-rotation of grain realigns the axes of grain and cavity, and (f) final state.

$$
\omega_{i j}^{\mathrm{lat}, g}=\Omega_{i j}+\tilde{\omega}_{i j}^{g}-\omega_{i j}^{p g}
$$

is the rotation rate of the crystallographic lattice associated with the grain.

Because of its definition in terms of the deviation with respect to the macroscopic spin, the polycrystal average of the ellipsoid spins fulfills the condition

$$
\left\langle\omega_{i j}^{g}\right\rangle=\Omega_{i j}
$$

Now let us analyze the case of two sites (identified as 1 and 2) interacting between them, as prescribed by the twosite VPSC model. Although algebraic convenience requires assigning ellipsoidal shapes to them, our intention is to use this configuration to describe the interaction across a boundary of two neighboring grains (or contiguous zones of two neighboring grains). And since we intend to enforce the corotation of the regions at each side of the boundary, we will describe them using flat ellipsoids whose axes have to remain parallel through deformation, as shown in Figure 6. In general, when using the two-site approach, the velocity gradients turn out to be different for each site after an incremental step. As a consequence,

$$
\begin{aligned}
\varepsilon_{i j}^{p, 1} & \neq \varepsilon_{i j}^{p, 2} \\
\omega_{i j}^{p, 1} & \neq \omega_{i j}^{p, 2} \\
\omega_{i j}^{1}=\Omega_{i j}+\tilde{\omega}_{i j}^{1} & \neq \Omega_{i j}+\tilde{\omega}_{i j}^{2}=\omega_{i j}^{2} \\
\omega_{i j}^{\mathrm{lat}, 1}=\Omega_{i j}+\tilde{\omega}_{i j}^{1}-\omega_{i j}^{p, 1} & \neq \Omega_{i j}+\tilde{\omega}_{i j}^{2}-\omega_{i j}^{p, 2}=\omega_{i j}^{\mathrm{lat}, 2}
\end{aligned}
$$

While the condition on the average rotation (Eq. [10]) is still fulfilled, Eq. [11] indicates that, in general, neither of the two ellipsoidal inclusions will co-rotate (their axes will not remain parallel), nor will the two associated crystal lattices co-rotate (their misorientation will not be preserved). If the two-site model describes the interaction across a grain boundary, one would intuitively expect that the latter two co-rotations would take place, to some extent, because of
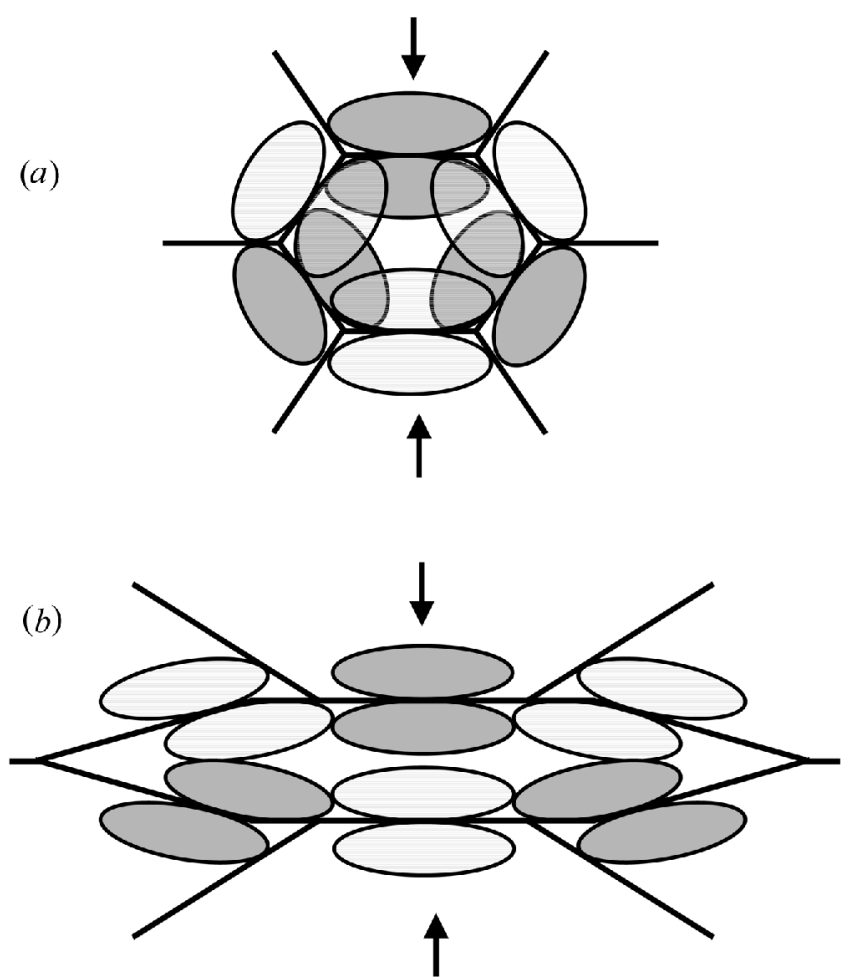

Fig. 6-Schematic 2-D representation of a grain with six neighbors as considered in a two-site VPSC simulation: (a) initial equiaxed configuration, and $(b)$ evolution of the grain morphology and of the set of ellipsoids during compression.

boundary constraints. As a consequence, we propose an effective rotation rate for the inclusions, where co-rotations are given by the average trend of both sites. We define

$$
\begin{aligned}
& \omega_{i j}^{\mathrm{eff}, 1}=\Omega_{i j}+\tilde{\omega}_{i j}^{1}+\Delta \omega_{i j}^{\mathrm{corot}} \\
& \omega_{i j}^{\mathrm{eff}, 2}=\Omega_{i j}+\tilde{\omega}_{i j}^{2}-\Delta \omega_{i j}^{\mathrm{corot}}
\end{aligned}
$$


and

$$
\begin{aligned}
& \omega_{i j}^{\mathrm{lat}, 1}=\omega_{i j}^{\mathrm{eff}, 1}-\omega_{i j}^{p, 1} \\
& \omega_{i j}^{\mathrm{lat}, 2}=\omega_{i j}^{\mathrm{eff}, 2}-\omega_{i j}^{p, 2}
\end{aligned}
$$

where

$$
\left.\Delta \omega_{i j}^{\mathrm{corot}}=\alpha_{i j}^{\mathrm{loc}} \frac{\tilde{\omega}_{i j}^{2}-\tilde{\omega}_{i j}^{1}}{2}-\alpha_{i j}^{p} \frac{\omega_{i j}^{p, 2}-\omega_{i j}^{p, 1}}{2} \quad \text { (no sum on } i j\right)
$$

Here, the parameters $\alpha_{i j}^{\text {loc }}$ and $\alpha_{i j}^{p}$ may, in principle, assume six independent values (i.e., $\alpha_{23}^{\text {loc }}, \alpha_{13}^{\text {loc }}$, and $\alpha_{12}^{\text {loc }} \alpha_{23}^{p}$, and $\alpha_{13}^{p}$, and $\alpha_{12}^{p}$ ). Observe that the previous definition guarantees that the average of the effective spins of the inclusions, given by Eq. [12], is the same as the average of the inclusion spins given by Eq. [11c]. As a consequence, the condition on the macroscopic average (Eq. [10]) is still fulfilled. There are three relevant limits and one particular case of Eq. [13], which we consider in what follows.

\section{Case in which $\alpha_{i j}^{\text {loc }}=\alpha_{i j}^{p}=0$, for all ij values}

This trivial case recovers the result of the formal twosite problem (Eq. [11]), which tends to misalign the two ellipsoids and also to modify the misorientation between both crystal lattices. As we discuss subsequently, textureand misorientation-evolution predictions made using this condition are not in good agreement with the experiment.

2. Case in which $\alpha_{i j}^{\text {loc }}=1$, and $\alpha_{i j}^{p}=0$ for all $i j$ values

This condition guarantees that the main axes of both ellipsoids co-rotate, but the lattices do not. In this case, the ellipsoid and crystal rotations are given by

$$
\begin{aligned}
& \omega_{i j}^{\mathrm{eff}, 1}=\omega_{i j}^{\mathrm{eff}, 2}=\Omega_{i j}+\frac{\tilde{\omega}_{i j}^{1}+\tilde{\omega}_{i j}^{2}}{2} \\
& \omega_{i j}^{\mathrm{lat}, 1}=\Omega_{i j}+\frac{\tilde{\omega}_{i j}^{1}+\tilde{\omega}_{i j}^{2}}{2}-\omega^{p, 1} \\
& \omega_{i j}^{\mathrm{lat}, 2}=\Omega_{i j}+\frac{\tilde{\omega}_{i j}^{1}+\tilde{\omega}_{i j}^{2}}{2}-\omega^{p, 2}
\end{aligned}
$$

Calculations done for the $\mathrm{Al}$ alloys using this assumption give minor differences in texture evolution and lateral anisotropy when compared with the aforementioned case 1 , corresponding to $\alpha_{i j}^{\text {loc }}=\alpha_{i j}^{p}=0$.

\section{Case in which $\alpha_{i j}^{\mathrm{loc}}=\alpha_{i j}^{p}=1$ for all ij values}

Under this condition, the lattice reorientation is the same for both crystals and, as a consequence, the lattices maintain their relative misorientation throughout deformation (they co-rotate). The main axes of the ellipsoids, however, do not co-rotate and become misaligned. In this case, the ellipsoid and crystal rotations are given by

$$
\begin{array}{r}
\omega_{i j}^{\mathrm{eff}, 1}=\Omega_{i j}+\frac{\tilde{\omega}_{i j}^{1}+\tilde{\omega}_{i j}^{2}}{2}+\frac{\omega_{i j}^{p, 1}-\omega_{i j}^{p, 2}}{2} \\
\omega_{i j}^{\mathrm{eff}, 2}=\Omega_{i j}+\frac{\tilde{\omega}_{i j}^{1}+\tilde{\omega}_{i j}^{2}}{2}-\frac{\omega_{i j}^{p, 1}-\omega_{i j}^{p, 2}}{2} \\
\omega_{i j}^{\mathrm{lat}, 1}=\omega_{i j}^{\mathrm{lat}, 2}=\Omega_{i j}+\frac{\tilde{\omega}_{i j}^{1}+\tilde{\omega}_{i j}^{2}}{2}-\frac{\omega_{i j}^{p, 1}+\omega_{i j}^{p, 2}}{2}
\end{array}
$$

The lattice co-rotation described by Eq. [15c] has been originally proposed by Bolmaro et al.,${ }^{[9]}$ using an argument based on a model of two inclusions fitting a single cavity. We find that the approach presented here provides a more rigorous way of looking at the problem and a clearer understanding of the approximations involved. According to Bolmaro and co-authors, the co-rotation scheme represented by Eq. $[15 \mathrm{c}]$ reproduces the deformation texture of twophase $\mathrm{Cu}-\mathrm{Fe}$ and $\mathrm{Ag}-\mathrm{Ni}$ composites ${ }^{[9]}$ and the observed evolution of small-angle misorientations in rolled Ag-Ni. ${ }^{[10]}$ However, the assumption of perfect co-rotation of the crystallographic lattices of two neighbor orientations may be too strict. In fact, Randle and Davies report that the misorientation between neighboring grains in deformed $\mathrm{Ni}^{[11]}$ and $\mathrm{Al}-\mathrm{Mg}^{[12]}$ is not completely preserved during deformation.

4. Case in which $\alpha_{23}^{\text {loc }}=\alpha_{23}^{p}=1 ; \alpha_{13}^{\text {loc }}=\alpha_{13}^{p}=1$; and $\alpha_{12}^{\text {loc }}=\alpha_{12}^{p}=0$

In this case, the spin tensors involved in the co-rotation scheme (Eq. [12]) have to be expressed in the reference frame $\left(x_{i}^{g}\right)$, defined by the three principal directions of the two interacting ellipsoids. The ellipsoid axes are parallel and the short axis $\left(x_{3}^{g}\right)$ is normal to the "grain boundary," represented here by the plane tangent to both ellipsoids (Figure 6). This condition is less restrictive than case 3, in that it does not enforce the full lattice co-rotation: the components $\omega_{13}^{\text {lat }}$ and $\omega_{23}^{\text {lat }}$ are the same for both crystals, but $\omega_{12}^{\text {lat }}$ is different. This amounts to a preservation of the parallelism of the crystallographic planes originally parallel to the tangent plane, while allowing a relative twist of the crystals around the normal-to-the-tangent plane. As for the ellipsoids, they do not co-rotate under the assumptions of this scheme. For calculation purposes, however, we enforce the parallelism of the ellipsoid pair after each step, while keeping invariant the crystallographic orientations updated through Eqs. [12c] and [d].

In what follows, we will adopt case 4 when considering co-rotation because, as we show in Section IV, the associated predictions are in better agreement with the in-plane anisotropy and the texture evolution observed in the three A1 alloys. Case 3 gives very similar results, but the evolution of misorientations is better captured by case 4 . In the onesite calculation, we may or may not account for co-rotation. When we do, then the orientations are paired at random. In the two-site calculation, we always include co-rotation, and the orientations are paired using the OIM information, as described in Section III-D. In all the cases involving corotation, the ellipsoids in each pair are assumed to be oblate and parallel to each other, with initial aspect ratios of 1:1:0.5. The orientations of the ellipsoidal pairs are assigned randomly.

\section{Hardening Law}

The experimentally determined true stress-true strain compression curves for the three $\mathrm{Al}$ alloys were used to adjust an extended Voce hardening law at the single-crystal level, of the form

$$
\tau_{(\Gamma)}=\tau_{0}+\left(\tau_{1}+\theta_{1} \Gamma\right)\left(1-\exp \left(-\frac{\theta_{0} \Gamma}{\tau_{1}}\right)\right)
$$

where $\tau$ is the critical resolved shear stress for $(111)\langle 110\rangle$ slip and $\Gamma$ is the accumulated shear in the grain. Deformation 
Table V. Single-Crystal Hardening Parameters (Equation [16]) That Fit the Experimental Compression Loading Data of Figure 1, for $\varepsilon_{\mathrm{ND}}=10^{-3}$ and for the Three Al Alloys (Units of MPa)

\begin{tabular}{lcrcc}
\hline Alloy & $\tau_{0}$ & $\tau_{1}$ & $\theta_{0}$ & $\theta_{1}$ \\
\hline AA5754 & 53 & 97 & 617 & 18 \\
AA5182 & 79 & 107 & 561 & 22 \\
AA6016 & 82 & 84 & 561 & 22 \\
\hline
\end{tabular}

was imposed in sequential incremental steps along the compressive direction, starting from the initial texture and reorienting the grains after each deformation step. In addition, the lateral stress components were enforced to be zero, and the evolution of the lateral strains was a consequence of this condition. The single-crystal hardening parameters that result from this fit are reported in Table $\mathrm{V}$ and were obtained by performing a one-site VPSC calculation, at an imposed macroscopic strain rate of $10^{-3} \mathrm{~s}^{-1}$ and using discrete texture files containing 2916 orientations derived from the X-ray measurements for the three Al alloys. The predicted loading curves are superimposed on the experimental ones in Figure 1. The agreement is within the dispersion of the experimental points when the compression tests are repeated. It was also verified that using OIM texture as initial input and accounting for correlations using the two-site VPSC models gives only small differences in the predicted loading curves. And since the final texture and lateral anisotropy are independent of the exact hardening law being used, the precision in fitting the loading curves is not an issue. The single-crystal hardening parameters that adjust the compression curve also reproduce the tensile case. The tensile loading curve predicted using those parameters and the one-site approach is depicted in Figure 2 for the AA5754 alloy. The predicted outof-plane anisotropy is discussed at the end of Section IV-B.

\section{Using OIM data as input}

In what follows, we describe how the OIM data are used as input of a two-site VPSC calculation. The OIM allows us to determine the orientation of each grain and that of the surrounding neighbors. We represent the interaction of a given grain with its neighbors using pairs of oblate ellipsoids, which, in reality, represent the grains' regions that are contiguous through the grain boundary. The ellipsoids associated with each pair are tangent, their axes are parallel, the short axes are co-linear, and the initial orientation of the ellipsoid axes is assigned at random. In our calculation, we use fully three-dimensional ellipsoid pairs. To illustrate the approach, Figure 6(a) shows a two-dimensional representation of an initially equiaxed grain with six neighbors. Six pairs of ellipsoids, each pair consisting of one ellipsoid having the measured crystallographic orientation of the central grain and another having the measured crystallographic orientation of one of its surrounding neighbors, represent all possible nearest-neighbor interactions for the grain at the center. After the polycrystal undergoes compression, the grain shape and the corresponding pairs of ellipsoids evolve to the configuration shown in Figure 6(b). The grain at the center is now represented by the six ellipsoids, which appear stretched and rotated after deformation and interaction with the nearest neighbors. The schematic representation of the morphologic texture (i.e., preferred orientation of the ellipsoid axes) is consistent with the actual evolution of the ellipsoid's orientation predicted by the VPSC model. Figure 7 shows the morphologic texture for AA5754 after 0, 25, and 50 pct compression, predicted with the two-site VPSC simulation. The pole-figure sequence shows the evolution of the ellipsoid's short-axis orientations, which tend to align with the compressive direction as deformation proceeds.

\section{RESULTS}

\section{A. Anisotropy Induced by Individual Rolling Components}

It is reasonable to regard the plastic response of the aggregate as a weighted average of the contributions arising from different grain orientations. As a consequence, separate simulations were performed on each of the rolling-texture components to determine the influence that each of them may have upon the in-plane anisotropy. For performing such calculations, a fictitious texture file comprising 200 orientations, each with a misorientation of, at most, 1 deg with respect to the ideal component, was generated. A uniaxial compression test along the ND was simulated using each of these "clusters" as the initial texture. Since we are dealing here with almost a single crystal, the kind of polycrystal model used (one-site or two-site) is irrelevant. Moreover, as we are only interested in the evolution of anisotropy (and not in the stress-strain response), the single-crystal hardening parameters used are not relevant either. The evolution in the ratio of the lateral strain components (along the RD and TD) is reported in Figure 8. The cube, rotated cube, brass, and Goss components are stable under compression. While the cube orientation keeps both lateral strains the same, rotated cube, brass, and Goss orientations overwhelmingly favor deformation along the RD. As a matter of fact, the rotated cube is not stable and slowly rotates into the Goss component. As a consequence, the contribution of these four components to the in-plane anisotropy will oppose the experimentally observed anisotropy. Copper and, to a lesser extent, the $\mathrm{S}$ component tend to give more deformation along the TD. However, both of them are unstable under axial compression, with $\mathrm{S}$ rotating towards the brass component and copper rotating away from any of the components analyzed here. As a consequence, the latter two components will counteract the effect on anisotropy of the other four only in the initial stages of the compression test, but, eventually, the reorientation will favor larger strain in the RD of the sheet. From the initial volume fractions listed in Table III, we can see that the two "competing" sets of components are in about the same proportion, and that they represent a smaller fraction than the "balance" component. As a consequence, although the results of this section are qualitatively useful, they do not allow us to predict the evolution of anisotropy in the aggregate, or even to figure out the overall trend. For this purpose, we need to simulate the deformation of the full aggregate.

\section{B. Predicted Textures and In-Plane Anisotropy}

Figure 9 shows the (111) pole figures corresponding to the compression textures of the AA5754 alloy, simulated with one-site/no co-rotation and two-site/co-rotation VPSC 


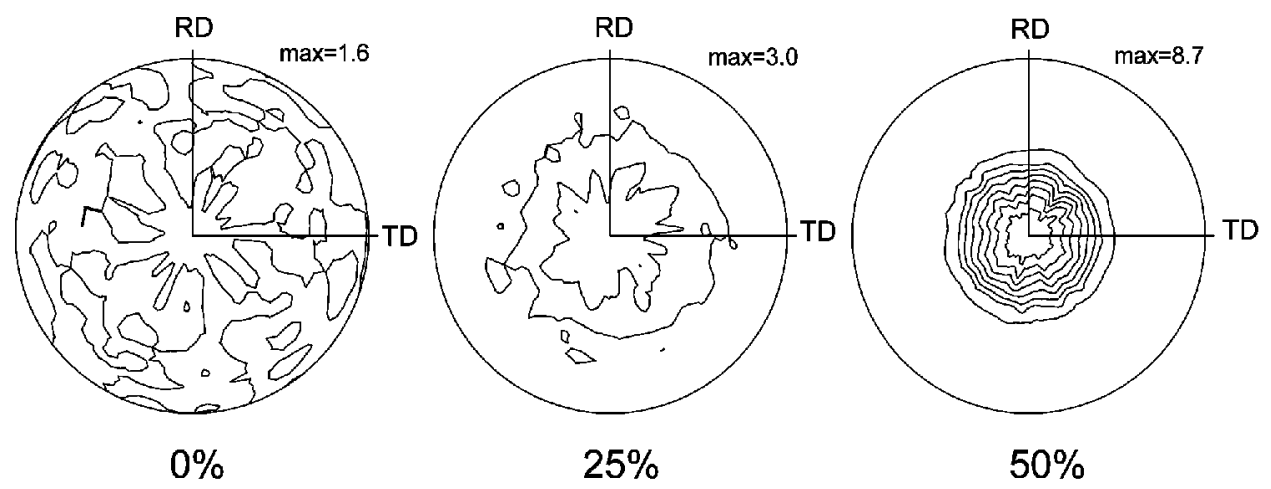

Fig. 7-Morphologic texture evolution during compression, represented as pole figures of the short axis of the ellipsoids. The short axis tends to align with the compressive direction (ND) as deformation proceeds.

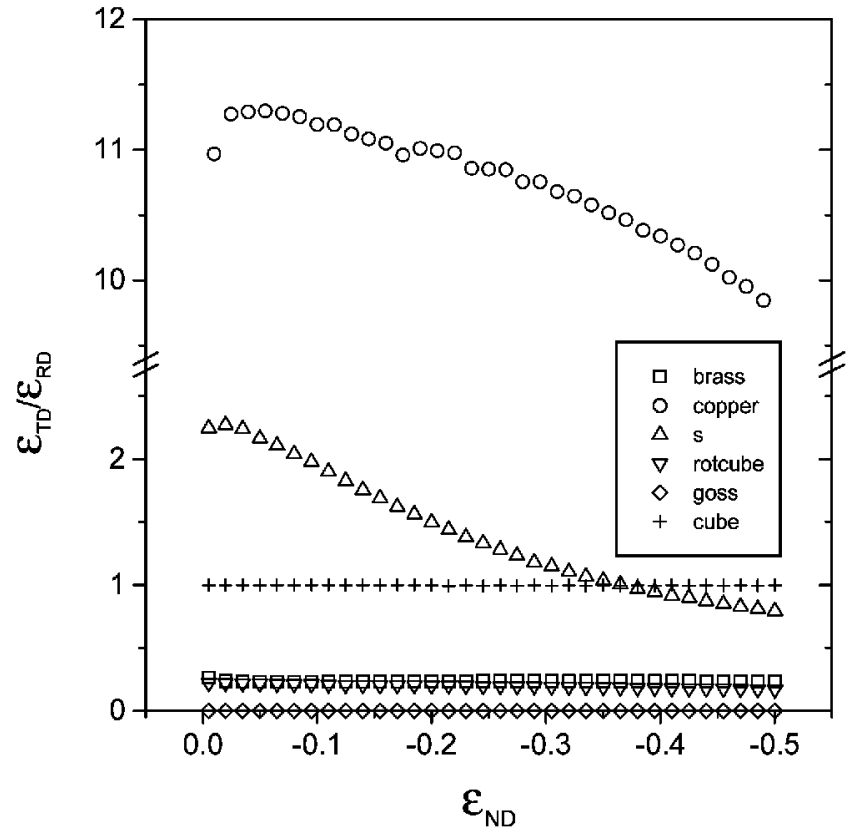

Fig. 8-Evolution of the lateral strain ratios for individual texture components during compression.

models, starting from the OIM initial texture. In the twosite case, the orientation pairing was done according to the OIM information (Sections II-C and III-D), allowing us to account for nearest-neighbor correlations. The one-site and two-site simulated textures are compared with the final texture, measured with X-rays (OIM measurements are not reliable for heavily cold-worked samples). While the textures are qualitatively similar, the intensity associated with the one-site/no co-rotation calculation is higher than the experimental one, indicating a faster texture evolution. The twosite/co-rotation approach, on the other hand, tends to evolve textures more slowly than than the one-site approach, which results in a better agreement with the experimental texture. An associated calculation shows that the short axes of the ellipsoids constituting the pairs tend to align with the compression direction during deformation (Figure 7). This rotation, which is given by Eq. [11c], increases as the eccentricity of the ellipsoidal inclusion increases and is also present in the one-site calculation. It indicates that both members of the pair tend to rotate in the same direction during compression, although not necessarily by the same amount.
A more revealing result is shown in Figure 10, where the evolution of the main rolling components during compression of AA5754 is compared for both approaches. As both simulations were started from the same texture, the initial fraction of each component starts the same, but they evolve differently. The arrows in Figure 10 indicate the final difference in volume fraction of each component, as predicted by the one-site and two-site models. The more relevant result is that, because the two-site approach slows the texture evolution, all the rolling components show a slower evolution. Judging from the discussion in Section IV-A about the role played by each component in the in-plane anisotropy, we conclude that the two-site results go "in the right direction" for explaining the in-plane anisotropy observed after $50 \mathrm{pct}$ compression. Specifically, the copper and S components, which favor higher lateral strains along TD but tend to diminish as compressive deformation proceeds, show a slower decrease in the two-site case. On the other hand, the brass and Goss components, which favor higher lateral strains along the RD and tend to increase with compressive deformation, show a less pronounced increase in the twosite case. As for the rotated cube (which also favors the TD strain), its proportion remains almost unaltered with deformation. Finally, the cube component also decreases less in the two-site than in the one-site case, but its variation does not affect the final ovalization.

The evolution of the rolling components obtained with both the one-site and two-site models for AA5754 is consistent with the predicted ovalization, shown in Figure 11 as the evolution of the ratio $\varepsilon_{T D} / \varepsilon_{R D}$ with comprehensive strain. Figure 11 shows results obtained with the one-site and twosite VPSC models, starting the simulation from the X-ray or the OIM initial texture. The figure also shows the measured values of $\varepsilon_{T D} / \varepsilon_{R D}$ corresponding to the final throughthickness compressive strain in each sample. A comparison with similar simulations done for the other two alloys, AA5182 and AA6016 (Figure 11), shows that, although the experimental in-plane anisotropy is qualitatively the same, AA5754 predictions exhibit features not present in the other two alloys.

According to Table III, AA5754 exhibits a much larger fraction of copper and $\mathrm{S}$ component than the other two alloys. As a consequence, the initial anisotropy is larger than for AA5182 and AA6016. However, the rapid evolution of the copper and $\mathrm{S}$ components associated with a one-site/no 

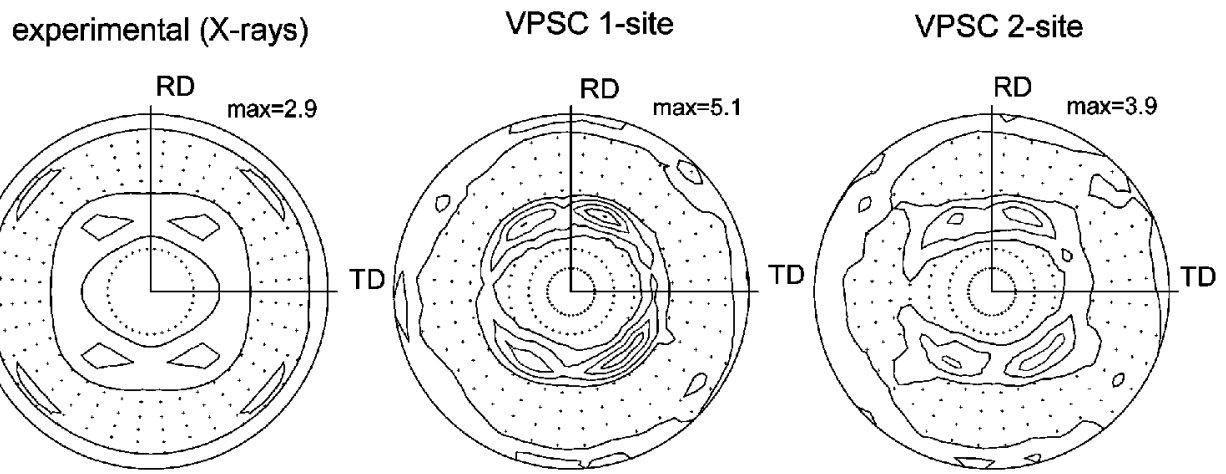

Fig. 9-(111) pole figures of the AA5754 Al alloy after 50 pct compression: (a) X-rays experimental texture, $(b)$ predicted texture using the one-site VPSC model (no corotation considered) starting from the initial OIM texture, and (c) predicted texture using the two-site VPSC model (with corotation), starting from the initial OIM texture. Lines are multiple of random distribution.

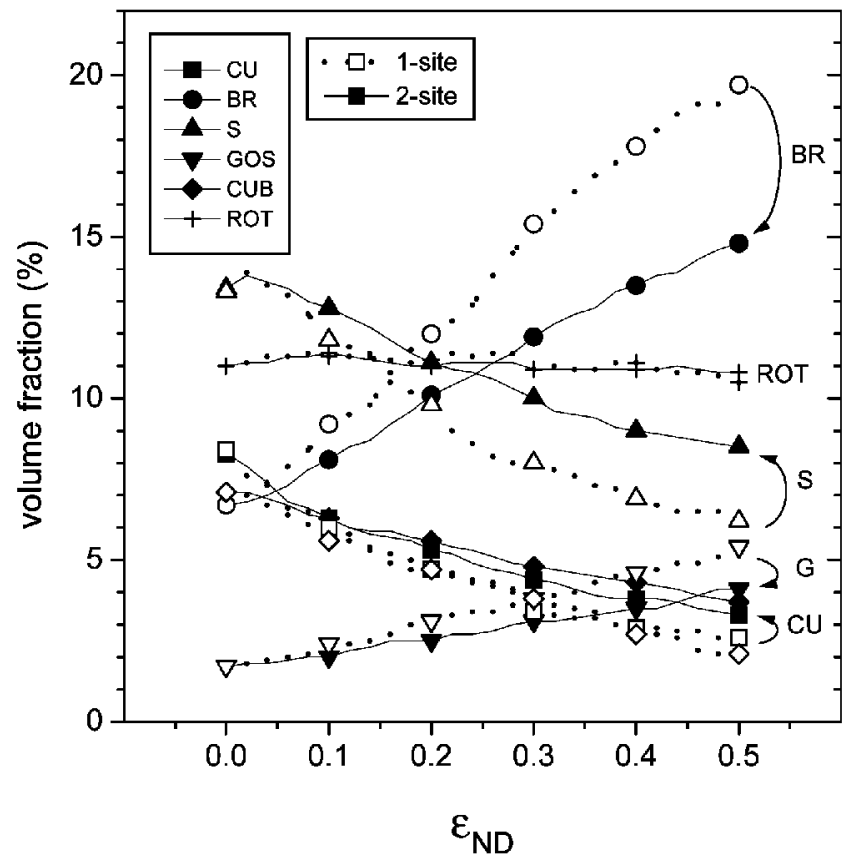

Fig. 10—Predicted evolution of rolling components of alloy AA5754 during compression simulations done using the one-site/no-corotation (open symbols) and two-site/corotation (solid symbols) approach and starting from the OIM texture.

co-rotation calculation tends to reverse the in-plane anisotropy after large strain. Such an effect is less pronounced for the two-site/co-rotation simulation, because of the slower texture evolution associated with it. Predictions done using the X-ray and the OIM textures show the same trend, but different absolute anisotropy (Figure 11). A possible explanation is that the in-plane anisotropy is very sensitive to details in the texture representation (Figure 3 ) and, correspondingly, to small differences in the proportion of retained rolling components (Table III). The predictions for AA5182 and AA6016 provide some evidence in this respect. While the one-site X-ray, one-site OIM, and two-site OIM predictions of anisotropy are very similar and consistent with the measured values (Figure 11), when orthotropic symmetry is forced into the X-ray texture, the predicted anisotropy drops substantially, although the texture is not far from orthotropic to start with (Figure 3). Since for AA5754 the symmetrized and unsymmetrized X-ray textures practically coincide (and predict the same anisotropy), we attribute the difference between the X-ray and OIM predictions to an artifact of the method used to determine the ODF from the X-ray measurements. Finally, a comparison of the two-site and the one-site results (both derived using the OIM texture) indicates that, as far as the in-plane anisotropy is concerned, there does not seem to be any particular advantage in using the more complicated two-site grain-interaction formulation. However, in what concerns the predicted deformation texture, we have seen that grain co-rotation schemes give more realistic texture intensities. As a consequence, grain corotation should be implemented in one-site or two-site models.

While for AA5754 the in-plane anisotropy is very sensitive to the details of the texture used, the out-of-plane anisotropy seems to be much less sensitive. Figure 12 depicts the experimental and predicted evolution of the lateral-strain components associated with a tensile test performed along the RD of the AA5754 sheet up to 15 pct strain. It is evident that the out-of-plane anisotropy is rather strong. In addition, the two "extreme" modeling conditions (one-site/no corotation/X-ray texture and two-site/co-rotation/OIM texture) give similar results in this particular case. In addition, the predictions are a good match to the measured strains, with the two-site approach being only slightly closer to them.

\section{Predicted Low- and High-Angle Misorientations}

The reliability of a model is frequently assessed through the amount of experimental information that the model can reproduce. In our particular case, using OIM data and corotation schemes allows us to introduce a new element of comparison between the experiment and model, in addition to the deformation texture and overall mechanical response. This new element provides grain-misorientation statistics. The OIM data used to represent the initial texture and to pair the orientations provides the initial grain-boundary crystallographic misorientations. Moreover, since the co-rotation scheme tracks the relative orientation of those pairs, it is also possible to predict changes in the high-angle misorientation distribution as deformation proceeds. Furthermore, by subdividing each grain into domains that may undergo different interactions and/or co-rotations (Figure 6), the present approach has associated with it a spread in the intragranular 

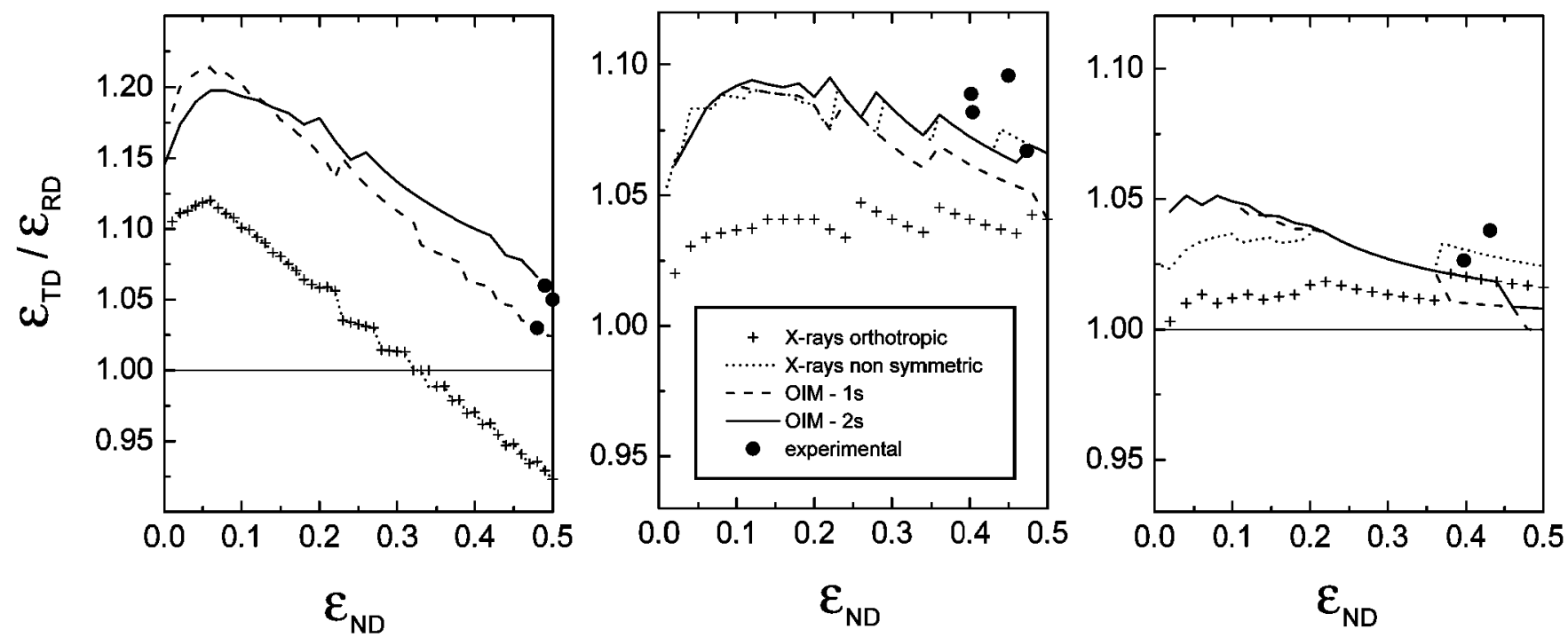

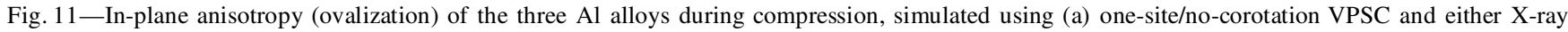

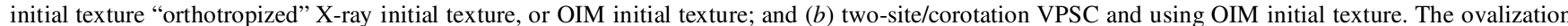
measured in various samples after compression is also shown for comparison (Table II).

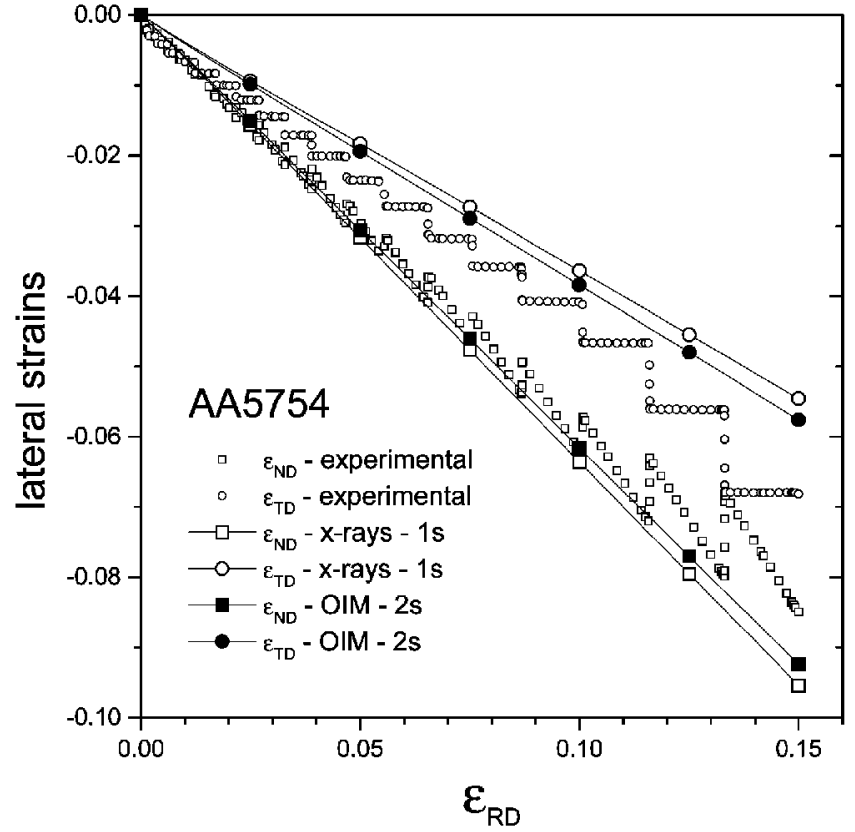

Fig. 12-Out-of-plane anisotropy of the alloy AA5754. Predicted and measured evolution of lateral strain components during a tensile test along the RD. Predictions correspond to (a) one-site VPSC, no corotation, X-ray initial texture; and $(b)$ two-site VPSC, with corotation and OIM initial texture

orientations, which can be loosely correlated with low-angle misorientation evolution and cell formation. We will show that low-angle and high-angle predicted misorientation distributions are consistent with the experimental evidence reported in the literature. Figure 13 shows the initial highangle misorientation distribution obtained by computing the misorientation angle between each orientation pair in the initial AA5754 texture. The distribution has the typical shape of the Mackenzie distribution, ${ }^{[13]}$ with a slightly higher mean value (42.8 deg) than the one associated with a random texture with no orientation correlations (i.e., $40.7 \mathrm{deg}$; refer to Reference 14). This slight difference is consistent with the mild texture and orientation correlations reported previously for these alloys. For completeness, Figure 13(a) also depicts the initial low-angle misorientation distribution as a single bar at $0 \mathrm{deg}$, indicating that, at the beginning, all the grain fragments have the same orientation. Figure 13(b) shows both distributions after 50 pct deformation, according to the two-site calculation. The low-angle misorientation distribution was obtained by computing the misorientation between each of the fragments associated with the same initial grain, but interacting with a different neighbor. The calculated curve is a typical lognormal distribution, i.e., the one usually reported in the literature. ${ }^{[15]}$ The overall highangle misorientation distribution, on the other hand, does not change much with respect to the initial one (the mean value increased $0.5 \mathrm{deg}$ ). In the latter case, however, we do not have the corresponding experimental information of high-angle misorientation, in the compressed sample. A more revealing result is obtained when computing the change in misorientation undergone by each individual pair of orientations, as shown in Figure 13(c). The calculated distribution exhibits an exponential decay, with about 50 pct of the pairs changing their misorientation by less than $2 \mathrm{deg}$. This result is consistent with recent measurements reported by Davies and Randle ${ }^{[11,12]}$ in fcc materials, which show a trend for neighboring grains to maintain compatibility across grain boundaries. Particularly, in the case of a 20 pct cold-rolled, initially recrystallized. Al-3 pct $\mathrm{Mg}$ alloy, ${ }^{[12]}$ these authors report a distribution of changes in misorientation ("misfit angles," in their terminology) with a profile similar to the one of Figure 13(c). Their measurements indicate that approximately 40 pct of grain boundaries change their misorientation by less than $2 \mathrm{deg}$, and the distribution shows an exponential decay with highest nonvanishing values at around $11 \mathrm{deg}$. 

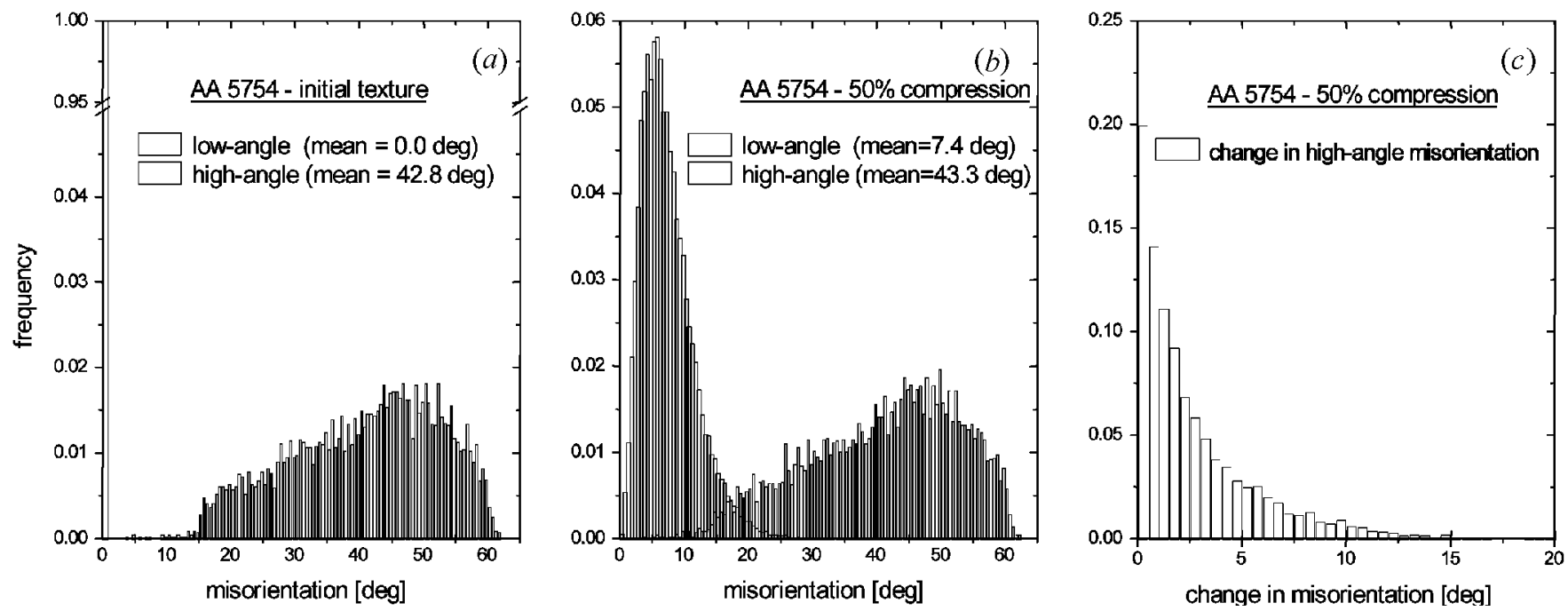

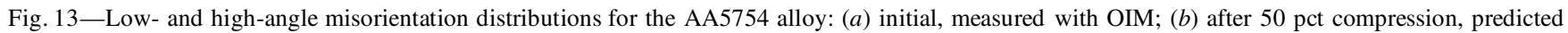

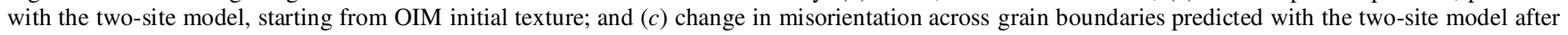
50 pct compression.

\section{CONCLUSIONS}

In this work, we do a comparative study of modeling approaches and their performance in reproducing the mechanical response, texture development, and crystallographic correlations of rolled and recrystallized aluminum. This work was originally motivated by our previous failure in reproducing the in-plane anisotropy observed in AA5754 alloys. ${ }^{[2]}$ Specifically, one-site simulations based on the initial texture measured by X-rays could not reproduce the consistently larger expansion along the TD observed in samples compressed along the ND. The added analysis of the AA5182 and AA6016 alloys allowed us to identify the texture representation as being responsible for the lack of agreement. The scope and the conclusions of this work, however, are of a more general character than the conclusion stated previously.

We explored possible spatial-correlation effects on the inplane anisotropy and, to such effect, we extended our model to include grain co-rotation and grain interaction during deformation. The fact that the various rolling and recrystallization components do not exhibit any systematic spatialorientation correlation in the three alloys analyzed here suggests that we discard the coupling between specific texture components as being responsible for the observed in-plane anisotropy. Rather, the fact that the evolution of the texture components is slower when grain co-rotations are accounted for indicates that this mechanism delays the evolution of texture, so preserving the in-plane anisotropy to larger strains. It is clear, from Figure 11, that eventually the accumulated anisotropy will be reversed in the AA5754 alloy for strains exceeding 50 pct. A grain co-rotation approach is appealing, because grains with the same initial orientation may reorient differently depending on the neighbor, and, second, the predicted deformation textures are not as sharp as those predicted by models without co-rotation. Both features are in better agreement with experimental evidence.

Different ways of accounting for grain interaction and grain co-rotation (or, equivalently, for grain cooperative deformation) within polycrystal models have been proposed in the past by several authors. Lee et al. ${ }^{[17]}$ have developed a model for shear banding in grains; Van Houtte et al. ${ }^{[18]}$ propose a so-called "lamel" model, which consists of enforcing the continuity of stress and strain components across the boundary of contiguous flat grains at random; Leffers ${ }^{[19]}$ proposes a variation of the lamel model in order to account for grain subdivision during rolling; Lebensohn and Canova $^{[4]}$ developed the two-site approach to simulate rolling of $\alpha+\beta$ Ti alloys; and Bolmaro et al. utilize grain co-rotations to simulate texture development of two-phase aggregates ${ }^{[9]}$ and evolution of misorientation during rolling of fcc polycrystals. ${ }^{[10]}$ What the aforementioned references have in common is that the enforcement of invariant crystallographic or invariant physical planes in grain pairs leads, indirectly, to grain interactions and, more directly, to grain co-rotation effects. In addition, the aforementioned references evidence an ongoing trend for incorporating microstructural mechanisms into polycrystal simulations.

One may wonder to what extent it is necessary to complicate polycrystal models in order to incorporate these concepts. There is not a unique answer to this question. Rather, the answer depends on how relevant some of these mechanisms may be in determining the material response which we are interested in simulating. In the particular case analyzed here, the out-of-plane anisotropy seems to be oblivious to details of the model used, while the in-plane anisotropy seems to be very sensitive to details in the texture representation. Along the same line, we observe that precise texture simulations require an accounting of the grain co-rotation effects, while grain-interaction effects play a lesser role. Aggregate hardening, on the other hand, is not sensitive to precise texture evolution, but it may be necessary to account explicitly for microstructure evolution in the grains. Introducing some of the improvements mentioned previously in hybrid applications that use a self-consistent polycrystal constitutive law inside finite-element codes ${ }^{[16]}$ may become computationally prohibitive. Our aim, in the latter case, is to simplify the approach while still capturing the relevant response of the aggregate.

Grain co-rotation considerations affect the evolution of low-angle misorientations, and a stronger connection 
between the experiments and model needs to be developed in this area. Here, we address the issue in a preliminary manner, mainly trying to make sure that our predictions do not violate available experimental information. While the result of such comparison is encouraging, we feel that a more systematic experimental and theoretical study will be required for elucidating the issue of co-rotations.

In what concerns the texture used in the model to represent the aggregate, we find that symmetrization of the X-ray texture affects the predicted in-plane anisotropy (Figure 11). Since OIM-based textures are less affected by numerical manipulation than X-ray textures, and since they contain information about crystallographic spatial correlations, we believe that OIM textures are better suited for simulation purposes.

As for the type of grain-interaction scheme to use, in the particular case of this study, the two-site ellipsoid interaction may be disregarded as a second-order effect, although it may be required when dealing with two-phase aggregates. ${ }^{[4]}$ Co-rotation or continuity conditions across the grain boundary (as used in References 18 and 19) seem to be necessary to enforce different reorientation paths in grains with the same initial orientation and, as a consequence, to predict a slower texture evolution with deformation. This requires us to assign ellipsoid orientations at random and enforce corotation of ellipsoidal pairs (Eq. [12]). The main disadvantage of this approach is computational, since the Eshelby tensor has to be calculated for each inclusion, but still, the one-site formulation has associated with it a simpler numerical algorithm than the two-site formulation.

\section{ACKNOWLEDGMENTS}

The authors thank Manuel Lovato for performing the compression tests and the Pechiney Centre de Recherches
(Voreppe, France) for providing the AA5162 and AA6016 $\mathrm{Al}$ sheets.

\section{REFERENCES}

1. U.F. Kocks and C.T. Necker: Proc. 15th Ris $\phi$ Int. Symp. on Materials Science, S.I. Anderson et al., eds., Ris $\emptyset$ National Laboratory, Roskilde, Denmark, 1994, pp. 45-58.

2. C.T. Necker, C.N. Tomé, F. Jean-Prost, and D.A. Korzekwa: Proc. 12th Int. Conf. on Textures of Materials (ICOTOM 12), J.A. Szpunar, ed., NRC Research Press, Ottawa, Canada, 1999, pp. 316-21.

3. R.A. Lebensohn and C.N. Tomé: Acta Metall. Mater. 1993, vol. 41, pp. 2611-24.

4. R.A. Lebensohn and G.R. Canova: Acta Mater., 1997, vol. 45, pp. 3687-94.

5. J.S. Kallend, U.F. Kocks, A.D. Rollett, and H.R. Wenk: Mater. Sci. Eng. A, 1991, vol. 132A, pp. 1-11.

6. S.I. Wright and B.L. Adams: Metall. Trans. A, 1992, vol. 23A, pp. 759-67.

7. R.A. Lebensohn, P.A. Turner, J.W. Signorelli, G.R. Canova, and C.N Tomé: Modelling Sim. Mater. Sci. Eng., 1998, vol. 6, pp. 447-65.

8. C.N. Tomé: Modelling Sim. Mater. Sci. Eng., 1999, vol. 7, pp. 723-38.

9. R.E. Bolmaro, R.A. Lebensohn, and H.-G. Brokmeier: Comp. Mater. Sci., 1997, vol. 9, pp. 237-50

10. R.E. Bolmaro, A. Fourty, A. Roatta, M.A. Bertinetti, P.A. Turner, and J.W. Signorelli: Scripta Mater, 2000, vol. 43, pp. 553-59.

11. V. Randle and R.K. Davies: Mater. Sci. Technol., 1999, vol. 15, pp. 750-54.

12. R.K. Davies and V. Randle: Mater. Sci. Eng. A, 2000, vol. 283A, pp. 251-65.

13. J.K. Mackenzie: Acta Metall., 1964, vol. 12, pp. 223-25.

14. M. Miodownik, A.W. Godfrey, E.A. Holm, and D.A. Hughes: Acta Mater., 1999, vol. 47, pp. 2661-68.

15. D.A. Hughes and N. Hansen: Acta Mater., 1997, vol. 45, pp. 3871-86.

16. C.N. Tomé, P.J. Maudlin, R.A. Lebensohn, and G.C. Kaschner: Acta Mater, 2001, vol. 49, pp. 3085-96.

17. C.S. Lee, B.J. Duggan, and R.E. Smallman: Acta Metall. Mater, 1993, vol. 41, pp. 2265-70.

18. P. Van Houtte, L. Delannay, and I. Samajdar: Text. Microstr., 1999, vol. 31 , p. 109.

19. T. Leffers: Int. J. Plasticity, 2001, vol. 17, pp. 491-511. 